\title{
A computer-controlled electrogustometer for the estimation of evoked taste thresholds
}

\author{
MALCOLM LOUDON and JENNIFER A. STILLMAN \\ Massey University, Auckland, New Zealand
}

\begin{abstract}
Reliable taste detection thresholds in humans are difficult to obtain for either research or clinical purposes because ordinary taste stimuli cannot be generated and presented by means of a computer. However, in investigations of taste dysfunction, electrical taste stimulation (electrogustometry) is sometimes employed. Minute anodal direct currents are applied manually to the tongue, giving rise to an acid taste experience through the liberation of protons into the saliva. This experience accords with recent findings on the mechanisms underlying sour taste perception. The device described here is a safe and accurate constant-current device, operating under computer control at very low amperages, making it possible to apply modern psychophysical procedures to the measurement of evoked taste thresholds.
\end{abstract}

Modern psychophysical procedures applied to the estimation of detection and difference thresholds in the sense of taste are subject to particular difficulties not encountered in the visual or auditory modalities, where the use of such procedures is routine. Suggestions for modifications in the methods generally adopted within food science for the measurement of difference thresholds have been made elsewhere (e.g., Stillman \& Irwin, 1995). In this paper, we describe a device that permits the estimation of taste detection thresholds by means of adaptive psychophysical procedures. Detection thresholds are useful in clinical investigations of human taste dysfunction, in the evaluation of treatment effectiveness, and in sensory research.

Multichannel computer-controlled stimulus delivery systems have been developed for psychophysical taste testing in animals. In particular, Brosvic and Slotnick (1986) developed an automated multichannel gustometer that they used with operant conditioning procedures to assess taste detection and discrimination in rats and to obtain measures of taste sensitivity in conjunction with a signal detection analysis (Brosvic \& Hecht, 1989). A multichannel computer-controlled gustometer for psychophysical taste testing in rats has also been developed by Spector, Andrews-Labenski, and Letterio (1990). By contrast, investigations of human taste sensitivity usually involve manual procedures such as the three-drop forced-choice method (see, e.g., Brosvic \& McLaughlin, 1989). This method is somewhat tedious and, particularly in cases of taste dysfunction, involves time-consuming trial-and-error to determine the appropriate range of tastant concentrations. However Krarup (1958) described a device for electrical taste stimulation that simplifies the measurement

The preparation of this manuscript was supported by a grant from the Massey University Research Fund to J.A.S. Correspondence should be addressed to J. A. Stillman, Department of Psychology, Massey University Albany, Private Bag 102-904, North Shore MSC, Auckland, New Zealand (e-mail: j.a.stillman@massey.ac.nz). of taste thresholds. The apparatus supplies very weak dc currents that do not stimulate the taste nerves directly, but produce ionic changes in the saliva and its constituents, which then become the taste stimulus. Usually anodal current, which liberates protons into the saliva, is applied, and thresholds are expressed in terms of current units, usually $\mu \mathrm{A}$. Anodal current elicits a sour (acid) taste experience, which accords with recent findings on the mechanisms underlying sour taste perception (McLaughlin \& Margolskee, 1994). By contrast, cathodal current elicits a soapy (alkaline) taste. Thresholds for cathodal taste are usually higher than those for anodal taste (Plattig, 1984).

Manual electrogustometry has been used in both research and clinical applications (Békésy, 1965; Hydén, Sandstedt, \& Ödkvist, 1982). Grant, Ferguson, Strang, Turner, and Bone (1987) investigated evoked taste thresholds in both a normal and a clinical population and judged $40 \mu \mathrm{A}$ to be the upper limit of normal when anodal current was used. Ajduković (1990) obtained thresholds averaging around $5 \mu \mathrm{A}$ from 6 volunteers of 19-25 years of age.

Response bias is a fundamental problem with the procedures used in conjunction with manual electrogustometry, because the sensory value adopted as a criterion for reporting that a taste experience occurred varies across individuals. However, because the stimulus is a galvanic one, electrogustometry has the potential to be used under computer control in conjunction with one of several adaptive forced-choice psychophysical procedures. Such procedures minimize bias and converge on a value of the stimulus for which the subject will attain a predetermined proportion of correct responses. For comparisons among a variety of candidate procedures see, for example, Hesse (1986); Shelton, Picardi, and Green (1982), and Stillman (1989).

Clearly the adoption of a computer-controlled electrogustometer for use with human subjects necessitates both primary and secondary isolation from the mains voltage and from the computer. We were unable to find a com- 
mercially available constant-current device operating at the very low values required for electrogustometry. In this paper, we provide details of the construction of an isolated electrogustometer that will deliver precise small amperages under computer control.

\section{OVERVIEW}

The electrogustometer comprises a programmable twochannel bidirectional voltage-controlled current supply with output currents in the range of $0-500 \mu \mathrm{A}$ in $1-\mu \mathrm{A}$ steps. All of its functions are controlled from an IBMPC-compatible computer via a serial link to the electrogustometer's internal microcontroller. The electrogustometer has no external controls and cannot be used as a stand-alone device. A Turbo Pascal unit contains the necessary driver software to interface between the Pascal programming language and the electrogustometer. Pascal procedures set the current through either of two channels, determine the current direction (anodal or cathodal), and measure the actual current flowing in a channel.

Upon receipt of a command from the PC to set the current, the electrogustometer's internal microcontroller (Dallas Semiconductor DS5000) consults a lookup table using the desired current as an index. The value thus obtained is translated into a voltage by a digital-to-analogue converter (DAC; Analogue Devices AD7549), the output of which drives the voltage-controlled current supply. The output current flows through the subject's body and through a precision sense resistor. From Ohm's law, the voltage developed across this resistor is directly proportional to the current flowing through the subject. The sensed voltage is then digitized by an analogue data acquisition unit (ADC) (Hitachi HD46509), referenced to a 5-V voltage reference (National Semiconductor LM336), and is fed back into the DS5000 microcontroller. The microcontroller compares the measured current with the desired current and increments or decrements the ADC as appropriate. The measure-compare-adjust feedback cycle is repeated indefinitely until a new command arrives for processing. By utilizing a closed loop system, the controlling PC can be alerted if the desired current cannot be driven through the subject, a circumstance that might occur in the event of poor electrode contact. The experimenter can thus be confident that the desired current is flowing.

The accuracy of the electrogustometer can be ascertained from Table 1, which compares desired current with measured current in each of its two channels. Measurements were made after the device was warmed up, using a Fluke 77 voltmeter and $0.1 \%$ current sense resistors. Measurements are shown for both anodal and cathodal current.

\section{ELECTROGUSTOMETER ELECTRONICS}

The electronics are contained on three circuit boards: the power supply board, the analogue board, and the digital board. These are described in turn.
Table 1

\begin{tabular}{|c|c|c|c|c|}
\hline \multirow{4}{*}{$\begin{array}{l}\text { Desired } \\
\text { Current }\end{array}$} & $\begin{array}{l}\text { ed Anodal } \\
\text { Measured } \\
\text { Each Ch }\end{array}$ & $\begin{array}{l}\text { odal or Ca } \\
\text { el of the } \mathrm{E} \text { I }\end{array}$ & $\begin{array}{l}\text { ent Compa } \\
\text { dal Curren } \\
\text { rogustomet }\end{array}$ & With \\
\hline & \multicolumn{4}{|c|}{ Measured Current } \\
\hline & \multicolumn{2}{|c|}{ Anodal } & \multicolumn{2}{|c|}{ Cathodal } \\
\hline & Channel 0 & Channel 1 & Channel 0 & Channel 1 \\
\hline 0 & 0.0 & 0.0 & 0.0 & 0.0 \\
\hline 1 & 0.9 & 0.9 & 1.0 & 0.9 \\
\hline 5 & 4.9 & 4.9 & 4.9 & 4.9 \\
\hline 10 & 9.9 & 9.9 & 9.9 & 9.9 \\
\hline 20 & 19.9 & 19.9 & 20.0 & 19.9 \\
\hline 30 & 29.9 & 29.9 & 29.9 & 29.9 \\
\hline 50 & 49.6 & 49.8 & 50.3 & 49.8 \\
\hline 60 & 59.8 & 60.0 & 60.4 & 60.0 \\
\hline 70 & 69.5 & 69.7 & 70.2 & 69.6 \\
\hline 80 & 79.9 & 80.1 & 80.6 & 80.1 \\
\hline 90 & 89.7 & 89.8 & 90.3 & 89.9 \\
\hline 100 & 99.9 & 99.9 & 100.5 & 99.8 \\
\hline 110 & 109.6 & 109.6 & 110.0 & 109.6 \\
\hline 120 & 119.9 & 120.1 & 120.5 & 120.0 \\
\hline 130 & 129.6 & 129.8 & 130.3 & 129.7 \\
\hline 140 & 139.8 & 139.9 & 140.3 & 139.9 \\
\hline 150 & 149.6 & 149.7 & 150.0 & 149.7 \\
\hline 200 & 199.8 & 200.1 & 200.6 & 200.1 \\
\hline 250 & 249.9 & 250.0 & 250.5 & 249.8 \\
\hline 300 & 299.6 & 299.7 & 300.3 & 299.6 \\
\hline 350 & 350.0 & 350.0 & 350.0 & 349.0 \\
\hline 400 & 399.0 & 399.0 & 400.0 & 399.0 \\
\hline 450 & 450.0 & 449.0 & 450.0 & 449.0 \\
\hline
\end{tabular}

Note-All currents are given in $\mu \mathrm{A}$.

\section{Power Supply Board}

The power supply board contains the isolated $\pm 15-\mathrm{V}$, $+5-\mathrm{V}$ power supply required to drive the remaining electronics and provide the double mains isolation required for equipment of this type, which is subject to regulations governing physiological applications. Primary mains isolation is provided by a $30-\mathrm{V}$ de plug pack that provides low voltage to the power supply board. The $30-\mathrm{V}$ dc is converted to $\pm 15 \mathrm{~V}$ and $+5 \mathrm{~V}$ by a dc-dc converter (SGS-Thomson GS5T24-5D15), which also provides secondary mains isolation $(2.5-\mathrm{kV} \mathrm{dc})$ in the event of catastrophic failure of the plug pack. It was found necessary to house the power supply board in a separate diecast box to prevent radio frequency $(\mathrm{RF})$ noise generated by the $\mathrm{dc}-\mathrm{dc}$ converter from interfering with the operation of the remaining electronics. Considerable lowpass filtering and shielding was required to reduce the $R F$ noise to an acceptable level. Future versions of the electrogustometer will use an alternative $\mathrm{dc}$ - dc conversion method to reduce the screening and filtering requirements.

\section{Digital Board}

The components of the digital board can be identified on the circuit diagram in Figure 1. These are the DS5000 microcontroller and associated clock and reset components; a dual DAC (AD7459) and associated operational amplifiers (LF353) used to convert the DAC's current output to a voltage; a 16-channel analogue data acquisition unit (HD46508), only two channels of which are used; a $5-\mathrm{V}$ voltage reference; and an optically isolated RS-232 serial interface. 


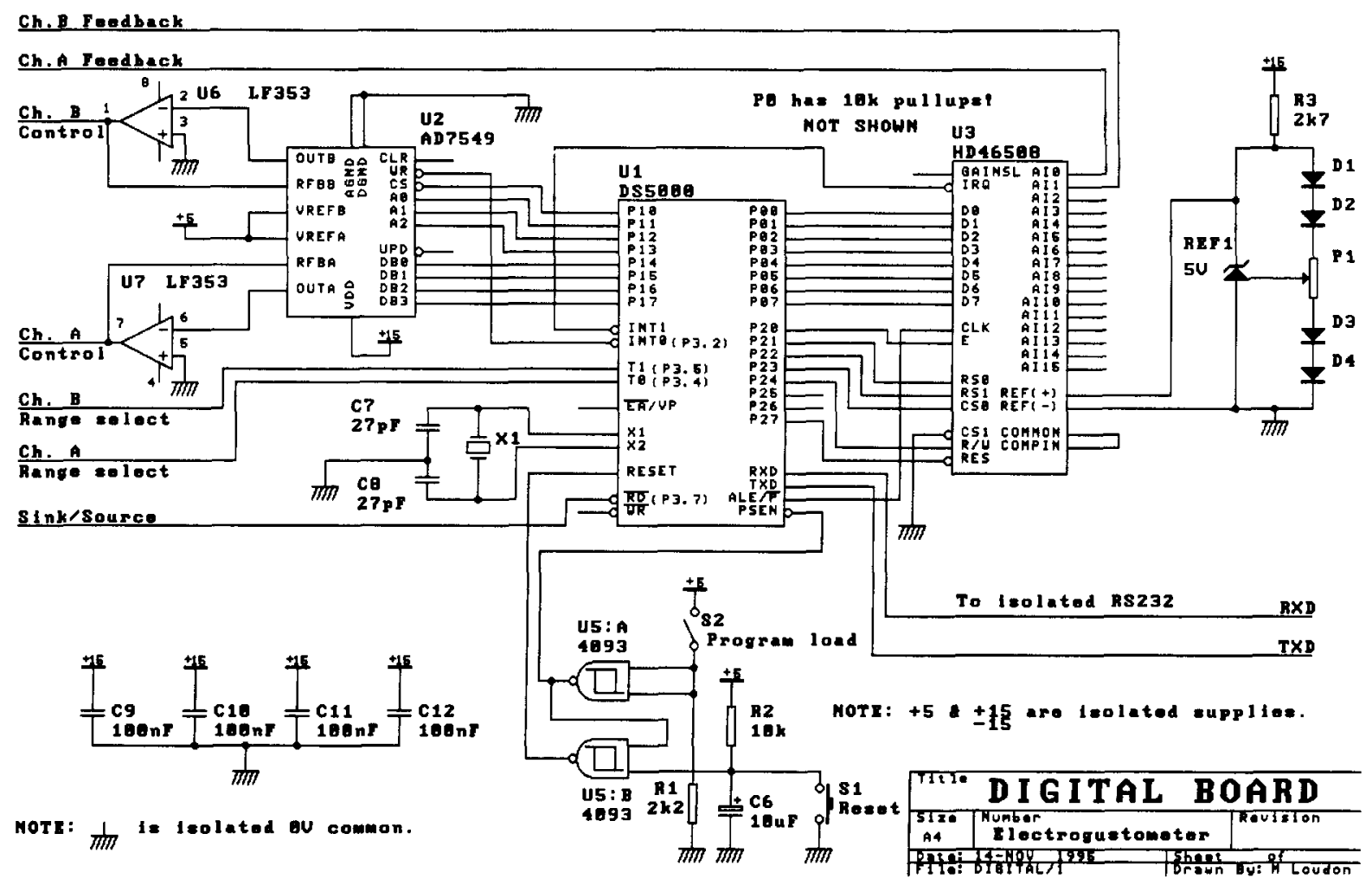

Figure 1. Schematic diagram of the electrogustometer digital board.

The DS5000 and associated reset circuitry warrant special mention. Unlike most embedded microcontrollers, the DS5000 contains no ROM as such. Instead it incorporates $32 \mathrm{~K}$ of nonvolatile RAM backed up by an onchip lithium battery providing memory for both program and data storage. Under normal operating conditions, the serial link is used to communicate commands to the electrogustometer and to receive data returned to the controlling PC. However, if the switch S2 is closed, and the Reset switch S1 is depressed, the DS5000 will enter "program load" mode. In this mode, normal operation is suspended, and the microcontroller will accept a new program communicated to it from the host computer down the serial link. This feature is especially useful during the early stages of software development when several minor, if not major, software changes are likely to be required. When a software change is needed, rather than the usual timeconsuming and cumbersome sequence of removing an EPROM or EPROM microcontroller, erasing the EPROM, burning in the new software, and reinstalling the EPROM, the new machine code is simply "squirted" down the serial link to the DS5000. The overall result is a dramatic reduction in embedded software development time, with the added bonus that new program features can be added quickly without the necessity of removing the DS5000 from its socket.

The AD7549 dual 12-bit DAC is connected in a conventional circuit to provide an independent control voltage for each of the voltage-controlled current supplies contained on the analogue board. The HD46508 is a flexible and economical 8/10-bit ADC with an on-chip 16-channel multiplexer and microprocessor interface. In this application, the HD46508 is set up to provide 10-bit measurements of the actual output current flowing from each of the two current sources.

To satisfy the double isolation requirements for mainsoperated physiological equipment, the serial interface between the electrogustometer and the controlling PC is optically isolated. The readily available $4 \mathrm{~N} 25$ opto-isolator is used for this purpose and provides $2.5-\mathrm{kV}$ dc isolation in the unlikely event that the host computer fails in such a manner that it presents mains voltages at the serial port. Primary insulation is thus provided by the host computer's power supply. The useful MAX232 chip forms the heart of the interface, generating the required RS-232 voltage levels from a single $+5-\mathrm{V}$ supply, thus considerably simplifying the interface.

\section{Analogue Board}

The analogue board contains two identical bipolar current supplies. Each supply consists of a voltage-controlled current source (OA4, Q2) and a voltage-controlled current sink (OA1, Q1) selected by RLY3. The microcontroller selects the current direction via the sink/source control line and Q3. To improve resolution at low currents, two ranges are available, selected by RLY 1 and RLY4. Range 1 provides $10 \mu \mathrm{A}$ per volt for low currents $(<50 \mu \mathrm{A})$, and Range 2 provides $100 \mu \mathrm{A}$ per volt for 
higher currents $(50-500 \mu \mathrm{A})$. The microcontroller selects the appropriate range via the range select control line and Q4. The remaining operational amplifiers provide voltage inversion (OA3, OA6) and buffering of the current sense resistors (OA2, OA5). The buffered voltage developed across the relevant current sense resistors, selected by RLY2, is fed back to the HD45608 via the feedback control line. The current sense resistors (R1, R2, $\mathrm{R} 5$, R6) have $0.1 \%$ precision to minimize current error due to resistor tolerance. All operational amplifiers connected across the sense resistors (OA1, OA2, OA4, and OA5) are MOSFET input devices (CA3 140) to minimize errors resulting from input bias currents. Figure 2 illustrates the negative control voltage version of the analogue board for one channel of the electrogustometer. The completed analogue board was dried in warm air and sprayed with a protective lacquer to prevent moisture ingress from causing leakage errors.

\section{OBTAINING THRESHOLD ESTIMATES}

The electrogustometer's two current channels connect to a pair of stainless steel active electrodes, each with a contact area of approximately $50 \mathrm{~mm}^{2}$. The electrodes are located on a specially designed holder that incorporates an adjustable chinrest and are movable in several planes. They unscrew for sterilizing. The electrode positions are adjusted for individual subjects so that each contacts one side of the tongue when it is slightly protruded and resting on the lower teeth. The tongue can be withdrawn into the mouth at any time. The indifferent electrode is a silver plate attached to the subject's wrist by means of a Velcro band.

The electrogustometer can be used with a variety of psychophysical procedures, depending on the purpose for which the data are collected. For example, a detectiontheory analysis could be applied to data obtained with the yes-no method, using only one channel of the electrogustometer to deliver a stimulus to the tongue. In our applications, however, threshold measures are obtained simultaneously from the left and right sides of the tongue using a two-alternative forced-choice (2AFC) method in conjunction with a modified adaptive staircase procedure targeted at $79.4 \%$ correct (Levitt, 1971). The events of a trial - warning interval, Observation Interval 1 , and Observation Interval 2-are depicted on the computer screen in the form of graphical representations of the actual warning and interval lights frequently used to mark the events of a trial with forced-choice psychophysical procedures. The analogue lights are "pulsed" on the screen in sequence with the events of a trial. The subject's task is to select the spatial and temporal interval that contained the taste stimulus. This is either the first observation interval and the left side of the tongue, or the second observation interval and the right side of the tongue. The current level is adjusted by the computer independently for

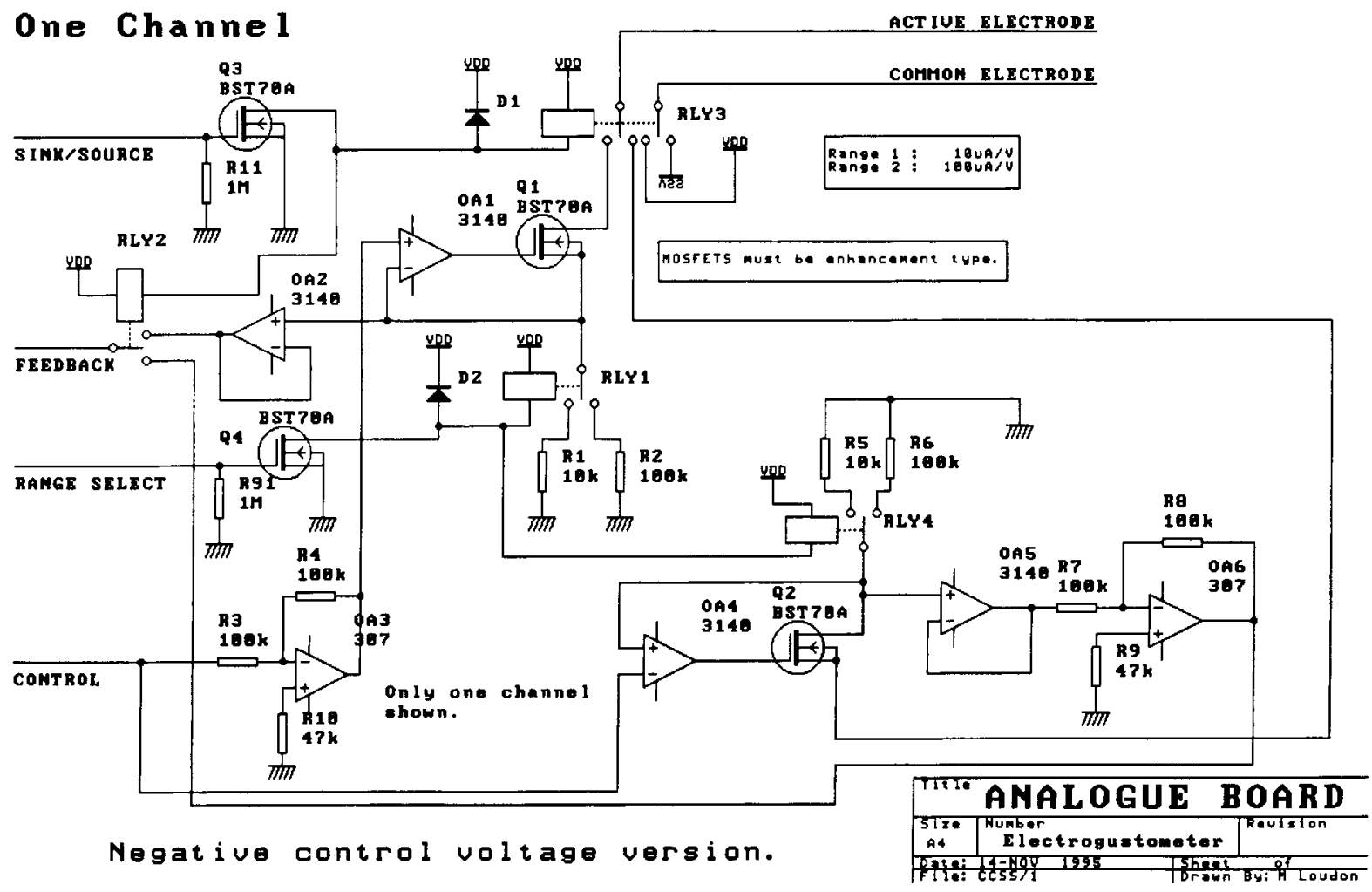

Figure 2. Schematic diagram of the analogue board for one channel of the electrogustometer. The diagram illustrates the negative control voltage version. 
left- and right-sided threshold runs in accordance with the rules of the staircase procedure. Current pulses are allocated randomly to either observation interval (and therefore to either side of the tongue) and constitute the basis of the forced-choice task with which the subject is confronted. A comparison between left- and right-sided thresholds is useful in investigations of taste dysfunction, and an advantage of this procedure is that pairs of estimates to be compared are obtained under identical experimental conditions.

A data record from 1 subject is shown in Figure 3. This figure illustrates several aspects of the psychophysical procedure we have used to obtain threshold estimates. With the staircase procedure targeted at $79.4 \%$ correct, the rules for changing the level of the stimulus specify a reduction following three correct responses and an increase following a single incorrect response. The reported threshold is the average of stimulus levels on trials at which the direction of change in the stimulus level reverses from increasing to decreasing or vice versa. The number of these "turnarounds" that contribute to a threshold estimate is decided in advance. In the trial block shown, the step size to the first reversal was $10 \mu \mathrm{A}$, and the step size during trials contributing to the final threshold estimate was $4 \mu \mathrm{A}$. Thresholds were the average of the eight turnarounds following the first two. The latter are considered settling values and do not contribute to the estimate. A consequence of the procedure used to obtain simultaneous thresholds from the two sides of the tongue is that additional trials will be presented to the side of the tongue that first reaches the specified number of turnarounds. This is because the two sides of the tongue correspond to the two spatial intervals of the 2AFC task, so that stimulus presentations must continue until each side has completed the designated number of turnarounds. In comparisons of thresholds for the two sides of the tongue, extra trials may be ignored in the calculation to ensure that pairs of threshold estimates are based on equal numbers of turnarounds.

Figure 3 also illustrates a consequence of the fact that the amperage required to elicit a taste experience in individuals with normal taste function is small. With the 2AFC method, a stimulus must be present in one or the other observation interval so that the stimulus cannot go to zero. Thus, since the electrogustometer operates in steps of $1 \mu \mathrm{A}$, a current of at least $1 \mu \mathrm{A}$ must be presented on each trial. On some occasions, an individual with normal taste function may make three correct choices at $1 \mu \mathrm{A}$, either by chance or as a result of genuine sensitivity. When this occurs, no further reductions in current are made, so that the actual threshold may be slightly lower than the calculated threshold. This floor effect is unlikely to be a problem in most clinical or research applications because very low thresholds will always be indicative of normal taste function. The initial presentation level, $31 \mu \mathrm{A}$ in this example, was designed to reach the $1-\mu \mathrm{A}$ limit in multiples of the initial step size $(10 \mu \mathrm{A})$; however, it is not necessary to follow this practice.

Finally, it may be preferable, when reporting electrical taste thresholds, to specify these in terms of current density $\left(\mu \mathrm{A} / \mathrm{mm}^{2}\right)$ rather than intensity, because there is evidence (Ajduković, 1990) that current density, rather than current intensity, is the actual taste stimulus.

\section{SUMMARY}

The matters discussed in this paper may be summarized as follows: The application of a small dc current to the tongue induces ionic changes in the saliva, which give rise to a taste experience that depends qualitatively on the direction of the current and quantitatively on the strength of the current. In individuals with normal taste function, very low amperages are required to reach detection threshold, particularly with anodal current.

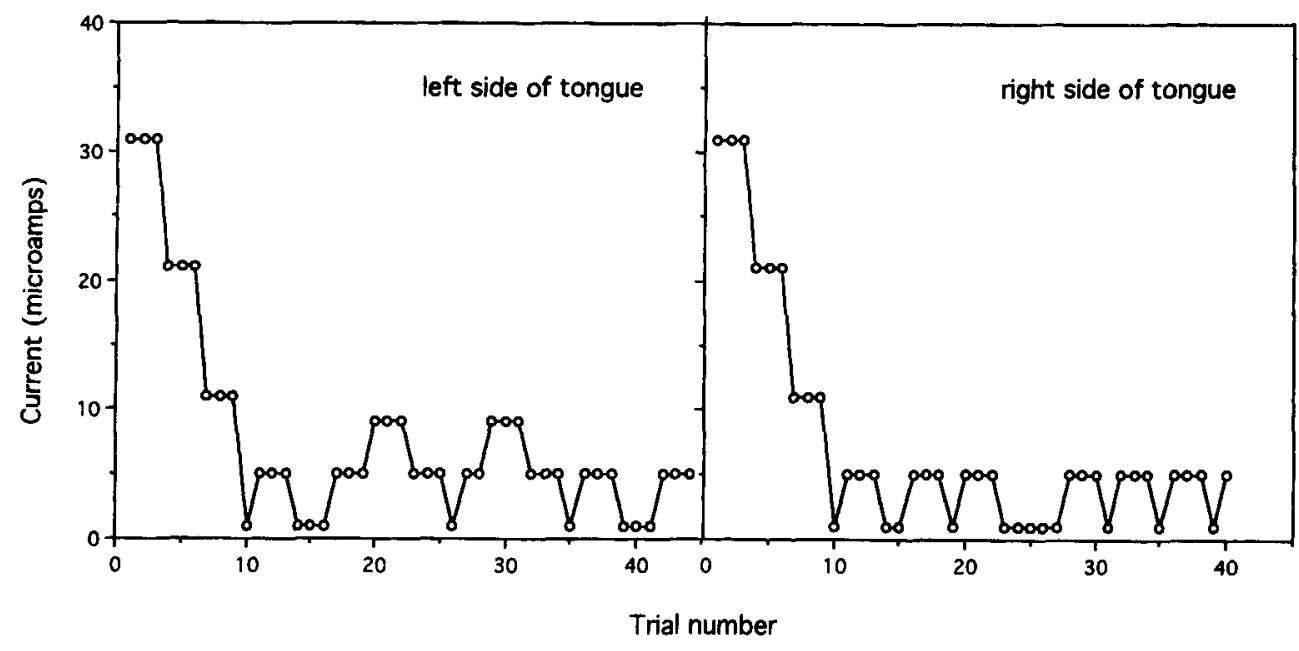

Figure 3. Example data record from 1 subject. The left- and right-sided thresholds were obtained concurrently. Left threshold $=4 \mu \mathrm{A}$ (current density $0.08 \mu \mathrm{A} / \mathrm{mm}^{2}$ ); right threshold $=3 \mu \mathrm{A}$ (current density $0.06 \mu \mathrm{A} / \mathrm{mm}^{2}$ ). 
Details have been given of the construction of an isolated electrogustometer that delivers the necessary precise low amperages under computer control. Such a device facilitates the application of adaptive psychophysical techniques to the study of human taste function. An adaptive version of the $2 \mathrm{AFC}$ procedure that estimates detection thresholds simultaneously from the left and right sides of the tongue was employed to illustrate the use of the electrogustometer.

The software that controls the device may be obtained from the authors on request.

\section{REFERENCES}

AJDUKović, D. (1990). Electrical taste stimulus: Current intensity or current density? Chemical Senses, 15, 341-347.

BÉKÉSY, G. voN (1965). Temperature coefficients of the electrical thresholds of taste sensations. Journal of General Physiology, 49, 27-35.

Brosvic, G. M., \& HECHT, G. S. (1989). Effects of copper and vitamin B-6 deficiency on taste sensitivity in the rat: A signal detection analysis. Physiology \& Behavior, 45, 1139-1146.

Brosvic, G. M., \& McLaughLin, W. W. (1989). Quality specific differences in human taste detection thresholds as a function of stimulus volume. Physiology \& Behavior, 45, 15-20.

Brosvic, G. M., \& SLotNick, B. M. (1986). Absolute and intensitydifference taste thresholds in the rat: Evaluation of an automated multichannel gustometer. Physiology \& Behavior, 38, 711-717.

Grant, R., Ferguson, M. M., Strang, R., Turner, J. W., \& Bone, I. (1987). Evoked taste thresholds in a normal population and the ap- plication of electrogustometry to trigeminal nerve disease, Journal of Neurology, Neurosurgery, \& Psychiatry, 50, 12-21.

HEsse, A. (1986). Comparison of several psychophysical procedures with respect to threshold estimates, reproducibility and efficiency. Acustica, 59, 263-273.

Hydén, D., SANDSTedt, P., \& Ödkvist, L.M. (1982). Prognosis in Bell's Palsy based on symptoms, signs and laboratory data. Acta Otolaryngologica, 93, 407-414.

KRARUP, B. (1958). Electrogustometry: A method for clinical taste examinations. Acta Otolaryngologica, 49, 294-305.

LEVITT, H. (1971). Transformed up - down methods in psychoacoustics. Journal of the Acoustical Society of America, 49, 467-477.

Mclaughlin, S., \& Margolskee, R. F. (1994). The sense of taste. American Scientist, 82, 538-545.

Plattig, K. H. (1984). The sense of taste. In J. R. Piggott (Ed.), Sensory analysis of foods (pp. 16-17). London: Elsevier.

Shelton, B. R., PiCardi, M. C., \& Green, D. M. (1982). Comparison of three adaptive psychophysical procedures. Journal of the Acoustical Society of America, 71, 1527-1533.

Spector, A. C., ANDrews-Labenski, J., \& Letterio, F. C. (1990). A new gustometer for psychophysical taste testing in the rat. Physiology \& Behavior, 47, 795-803.

StILlman, J. A. (1989). A comparison of three adaptive psychophysical procedures using inexperienced listeners. Perception \& Psychophysics, 46, 345-350.

StILlman, J. A., \& IRWIN, R. J. (1995). Advantages of the same-different method over the triangular method for the measurement of taste discrimination. Journal of Sensory Studies, 10, 261-272.

(Manuscript received December 18, 1995; revision accepted for publication July 3,1996 .) 